\title{
BEAM DYNAMICS DESIGN OF THE L3BT FOR J-PARC
}

\author{
T. Ohkawa ${ }^{\#}$, JAERI, Ibaraki, Japan \\ M. Ikegami, KEK, Ibaraki, Japan
}

\begin{abstract}
L3BT is beam transportation line from the linac to the 3-GeV RCS which is the part of the accelerators for the High-Intensity Proton Accelerator Facility Project, JPARC. In this paper, results of the beam simulation of the injection section of the L3BT are presented. The injection section is necessary for a successful adjustment of the transported beam to the required parameters for ring injection. The matching of rms envelopes and dispersion function for high-intensity beams are also discussed.
\end{abstract}

\section{INTRODUCTION}

The accelerators for J-PARC consist of a $180-\mathrm{MeV}$ linac, a 3-GeV RCS (Rapid Cycling Synchrotron), and a 50-GeV MR (Main Ring) [1].

To meet the requirement for the beam loss minimization, the L3BT does not only connect the linac to the $3 \mathrm{GeV}$ RCS, but also modifies the linac beam to be acceptable for the RCS. The required beam parameters at the injection point of the RCS are

Momentum spread $< \pm 0.1 \%(100 \%)$ and

Transverse emittance $<4 \pi \mathrm{mm} * \operatorname{mrad}(100 \%)$.

To achieve these beam qualities, the L3BT should have following functions: momentum compaction, transverse halo scraping and beam diagnosis.

The L3BT consists of a straight section, an achromatic arc section, a scraper section and an injection section. Two debunchers are set up in the L3BT. The debunchers are used to obtain the momentum spread of less than \pm $0.1 \%$ at the injection point of the RCS. Another effect of the debunchers is the energy centroid correction when the beam energy is shifted from the design value due to RF errors in the DTL (Drift Tube Linac) and SDTL (Separate-type DTL) section. The vertical bump magnet for the vertical painting injection is placed at a position of $\pi$ phase difference from the injection point.

\section{PRECONDITION}

PARMILA code is used for the beam simulation. The quadruple focusing strengths are determined by using TRACE3D. TRACE3D is the envelope analysis code including the space-charge effect and it has the parameter matching function [2][3].

The dispersion function calculated in TRACE3D characterizes the beam width broadening due to momentum spread, which is generally different from the central orbit distortion due to momentum deviation. At the center of the beam distribution the space charge force is cancelled and the dispersion function is the same as the case when the beam current is $0 \mathrm{~mA}$. We calculate two

\footnotetext{
\#tohkawa@linac.tokai.jaeri.go.jp
}

dispersion function for the same lattice with and without space charge and call the former "dispersion function R" and the latter "dispersion function C". The dispersion function $\mathrm{R}$ means the derivatives of the rms envelope with respect to momentum $\mathrm{p}$ and the dispersion function $\mathrm{C}$ means the derivatives of the central orbit with respect to momentum $\mathrm{p}$. When the beam current is $0 \mathrm{~mA}$ two types of dispersion functions is the same.

\section{BEAM SIMULATION}

We calculate two types of optics for the injection section of the L3BT as follows: The dispersion function $\mathrm{R}$ at the injection point of the RCS is 0 in one case (case 1) and the dispersion function $\mathrm{C}$ at the injection point of the RCS is 0 in the other (case 2).

At first, the envelope analyses of the L3BT are performed with TRACE3D for above two cases. The calculation results are shown in Fig. 1 and Fig. 2. The beam parameters at the RCS injection point are shown in Table 1.

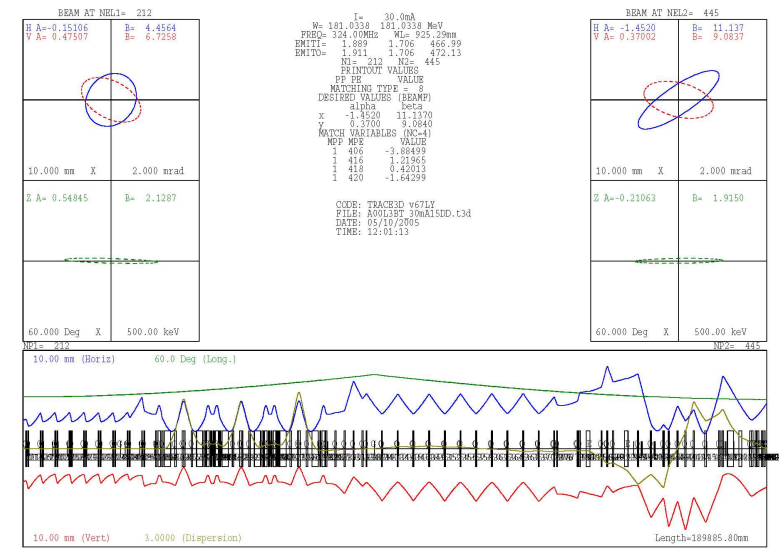

Figure 1: Beam envelope of the L3BT (case 1).

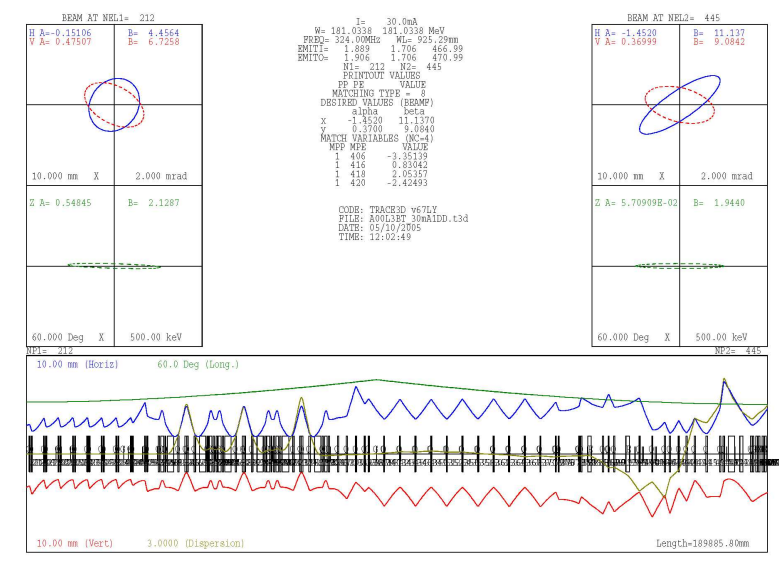

Figure 2: Beam envelope of the L3BT (case 2). 
Table 1: Beam parameters at the RCS injection point

\begin{tabular}{cccc}
\hline & Unit & Case 1 & Case 2 \\
\hline \hline $\mathrm{E}$ & $\mathrm{MeV}$ & 181.034 & 181.034 \\
$\alpha \mathrm{x}$ & & -1.452 & -1.452 \\
$\beta_{\mathrm{x}}$ & $\mathrm{m}$ & 11.137 & 11.137 \\
$\beta_{\mathrm{x} \text { max }}$ & $\mathrm{m}$ & 36.718 & 28.037 \\
$\beta_{\mathrm{x} \min }$ & $\mathrm{m}$ & 1.209 & 2.224 \\
$\eta_{\mathrm{RX}}$ & $\mathrm{m}$ & 0.0 & -1.476 \\
$\eta_{\mathrm{RX}}^{\prime}$ & & 0.037 & -0.136 \\
$\eta_{\mathrm{CX}}$ & $\mathrm{m}$ & -0.950 & 0.0 \\
$\eta_{\mathrm{CX}}$ & & -0.083 & 0.0 \\
$\alpha \mathrm{y}$ & & 0.370 & 0.370 \\
$\beta_{\mathrm{y}}$ & $\mathrm{m}$ & 9.084 & 9.084 \\
$\beta_{\mathrm{y} \max }$ & $\mathrm{m}$ & 34.711 & 21.265 \\
$\beta_{\mathrm{y} \min }$ & $\mathrm{m}$ & 3.407 & 3.428 \\
$\alpha \mathrm{Z}$ & & -0.211 & 0.057 \\
$\beta \mathrm{z}$ & $\operatorname{deg}^{*} \mathrm{MeV}$ & 1915 & 1944 \\
$\Delta \mathrm{p} / \mathrm{p}$ & $\%$ & 0.0022 & 0.0021 \\
\hline
\end{tabular}

Table 1 shows that the maximum values of $\beta \mathrm{x}$ and $\beta y$ of case 1 are larger than those of case 2 . In case 2 , the variation of the twiss parameters due to the space-charge effects is small.

Next, the simulations from the MEBT to the RCS injection point are performed with PARMILA. Assumed peak current is $30 \mathrm{~mA}$ and a $3 \mathrm{D}$ space-charge routine is adopted. The beam distribution based on the experiment and simulation result of RFQ is used as the initial beam distribution at the entrance of the MEBT [4]. Initial parameters are shown in Table 2. The calculation results of the beam parameters at the RCS injection point are shown in Table 3.

Table 2: Initial parameters at the MEBT entrance

\begin{tabular}{lc}
\hline Number of particles & 95322 particles \\
$\varepsilon \mathrm{x}_{0}(\mathrm{rms})$ & $0.212 \pi \mathrm{mm} * \mathrm{mrad}$ \\
$\varepsilon \mathrm{y}_{0}(\mathrm{rms})$ & $0.212 \pi \mathrm{mm}^{*} \mathrm{mrad}$ \\
$\varepsilon \mathrm{z}_{0}(\mathrm{rms})$ & $0.091 \pi \mathrm{MeV}^{*} \mathrm{deg}$ \\
$\varepsilon \mathrm{x}_{0}(99.5 \%)$ & $2.08 \pi \mathrm{mm}^{*} \mathrm{mrad}$ \\
$\varepsilon \mathrm{y}_{0}(99.5 \%)$ & $2.05 \pi \mathrm{mm} * \mathrm{mrad}$ \\
$\varepsilon \mathrm{z}_{0}(99.5 \%)$ & $1.32 \pi \mathrm{MeV}^{*} \mathrm{deg}$ \\
\hline
\end{tabular}

Table 3: Beam parameters at the RCS injection point

\begin{tabular}{cccc}
\hline & Unit & Case 1 & Case 2 \\
\hline \hline $\mathrm{E}$ & $\mathrm{MeV}$ & 181.034 & 181.034 \\
$\alpha \mathrm{x}$ & & -1.617 & -1.478 \\
$\beta \mathrm{x}$ & $\mathrm{m}$ & 10.589 & 10.009 \\
$\Delta \mathrm{x}_{\mathrm{c}}$ & $\mathrm{mm}$ & 0.046 & 0.002 \\
$\varepsilon \mathrm{x} / \pi$ & $\mathrm{mm} \mathrm{m}^{*} \mathrm{mrad}$ & 4.320 & 3.975 \\
$\alpha \mathrm{y}$ & & 0.442 & 0.368 \\
$\beta \mathrm{y}$ & $\mathrm{m}$ & 9.750 & 8.934 \\
$\varepsilon \mathrm{y} / \pi$ & $\mathrm{mm}^{*} \mathrm{mrad}$ & 3.647 & 3.469 \\
$\alpha \mathrm{z}$ & & -0.774 & -0.669 \\
$\beta \mathrm{z}$ & $\mathrm{deg}^{*} \mathrm{MeV}$ & 742.7 & 755.3 \\
$\varepsilon \mathrm{z} / \pi$ & $\mathrm{MeV}^{*} \mathrm{deg}$ & 4.105 & 4.068 \\
$\Delta \mathrm{p} / \mathrm{p}$ & $\%$ & 0.025 & 0.024 \\
\hline
\end{tabular}

In Table 3, $\varepsilon x$ and $\varepsilon y, \varepsilon z$ denote $99.5 \%$ transverse and longitudinal emittance, respectively. $\Delta \mathrm{x}_{\mathrm{c}}$ is the distortion of the central orbit and $\Delta \mathrm{p} / \mathrm{p}$ is the momentum spread at the injection point which is calculated by using $99.5 \%$ emittance and $\beta \mathrm{x}$. In the two cases, the momentum spread at the injection point of the RCS is almost the same, which satisfies the requirement of less than $\pm 0.1 \%$. Table 3 shows that $99.5 \%$ emittance of case 1 is larger than that of case 2 .

We conclude from Table 1 and 3 that case 2 is more stable for the space-charge effects than case 1 .

\section{RF Errors}

The simulations from the MEBT to the RCS injection point are performed assuming RF errors in the DTL and SDTL. A peak beam current is $30 \mathrm{~mA}$ and space charge effect is calculated with three dimensions. The same beam distribution shown in Table 2 is used as initial beam distribution at the entrance of the MEBT.

The RF field amplitude and phase errors for each tank of the DTL and the SDTL are randomly generated within the range of $\pm 1 \%$ and $\pm 1 \mathrm{deg}$. In addition, it is assumed that there are 6 deg phase and $6 \%$ amplitude tuning errors for the debunchers. We shall concentrate on the worst case that the energy at the end of the SDTL is maximum $(\Delta \mathrm{W}=0.410 \mathrm{MeV})$. [5] The calculation results of the energy and beam parameters at the RCS injection point are shown in Table 4.

Table 4: Simulation results of the beam parameters

\begin{tabular}{cccc}
\hline & Unit & Case 1 & Case 2 \\
\hline \hline $\mathrm{E}_{\text {ref }}$ & $\mathrm{MeV}$ & 181.034 & 181.034 \\
$\mathrm{E}_{\mathrm{SDTL}}$ & $\mathrm{MeV}$ & 181.445 & 181.445 \\
$\alpha \mathrm{x}$ & & -1.802 & -1.579 \\
$\beta \mathrm{x}$ & $\mathrm{m}$ & 11.130 & 10.328 \\
$\Delta \mathrm{x}_{\mathrm{c}}$ & $\mathrm{mm}$ & 0.672 & 0.003 \\
$\varepsilon \mathrm{x} / \pi$ & $\mathrm{mm}{ }^{*} \mathrm{mrad}$ & 4.132 & 3.934 \\
$\alpha \mathrm{y}$ & & 0.514 & 0.403 \\
$\beta \mathrm{y}$ & $\mathrm{m}$ & 11.284 & 9.319 \\
$\varepsilon \mathrm{y} / \pi$ & $\mathrm{mm}^{*} \mathrm{mrad}$ & 3.542 & 3.452 \\
$\alpha \mathrm{z}$ & & -1.425 & -1.443 \\
$\beta \mathrm{z}$ & $\mathrm{deg}{ }^{*} \mathrm{MeV}$ & 657.2 & 683.0 \\
$\varepsilon \mathrm{z} / \pi$ & $\mathrm{MeV} \mathrm{deg}$ & 23.023 & 22.569 \\
$\mathrm{E}_{\mathrm{RCS}}$ & $\mathrm{MeV}$ & 180.795 & 180.795 \\
$\delta$ & $\%$ & -0.072 & -0.072 \\
$\Delta \mathrm{p} / \mathrm{p}$ & $\%$ & 0.041 & 0.040 \\
\hline
\end{tabular}

In Table $4, \mathrm{E}_{\text {ref }}, \mathrm{E}_{\mathrm{SDTL}}$, and $\mathrm{E}_{\mathrm{RCS}}$ denote the design energy, output energy of SDTL, and the energy at the injection point, respectively. $\delta$ is the momentum difference divided by the reference momentum.

Table 4 shows that even if the dispersion function $\mathrm{R}$ is 0 the central orbit is shifted. The distortion of the central orbit is almost consistent with the value that is calculated by using the dispersion function $\mathrm{C}$ in the Table 1 .

We conclude from Table 1, 3 and 4 that case 2 (the derivatives of the central orbit with respect to momentum 
$\mathrm{p}$ is 0 ) is better than case 1 (the derivatives of the rms envelope with respect to momentum $p$ is 0 ). The beam distributions at the injection point of the RCS are shown in Fig. 3 and Fig. 4.

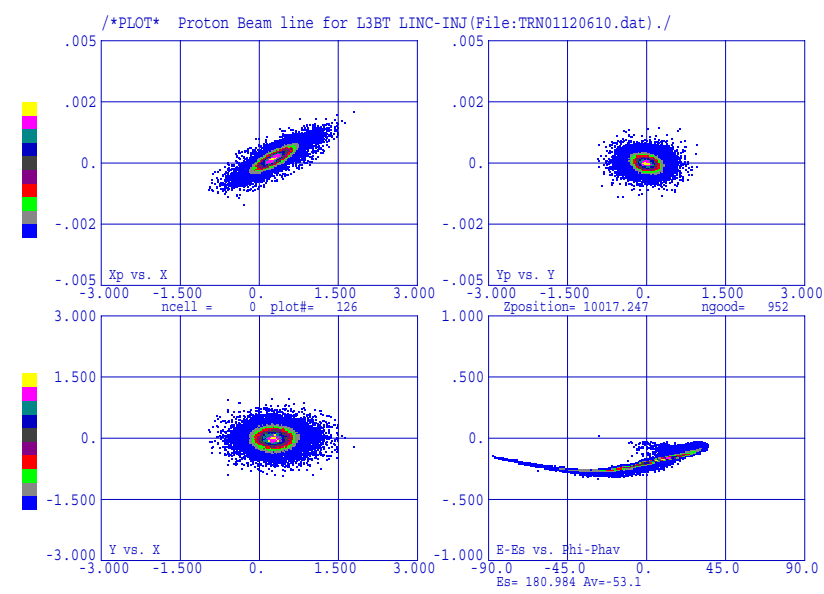

Figure 3: Beam distribution at the injection point of the RCS (case 1).

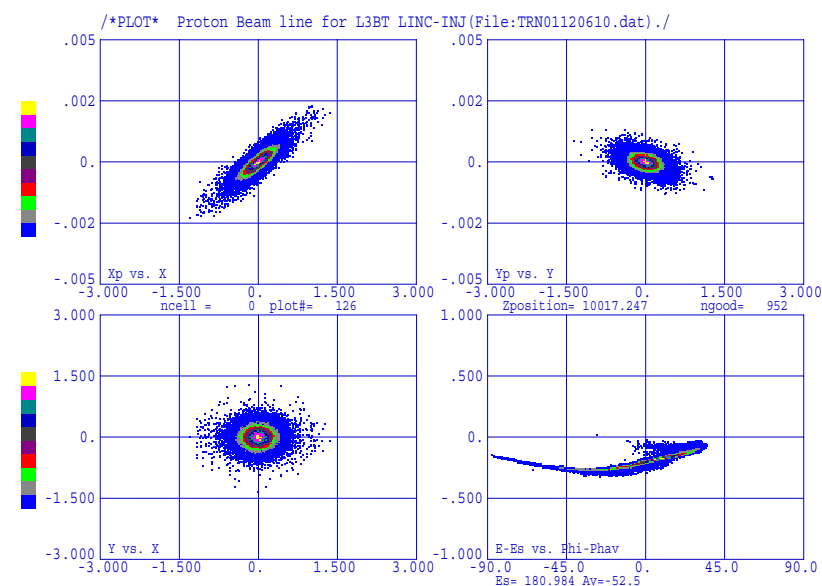

Figure 4: Beam distribution at the injection point of the RCS (case 2).

\section{SUMMARY}

We calculate two types of optics for the injection section of the L3BT, which is different in the optimisation of the dispersion function. In one case, we have optimised to eliminate the zero-current dispersion at the RCS injection point, whereas we eliminate the dispersion with space-charge in the other. We have confirmed that we should suppress the zero-current dispersion in our case to avoid excess emittance growth.

\section{REFERENCES}

[1] Y. Yamazaki(eds), "Accelerator Technical Design Report for J-PARC”, KEK-Report 2002-13; JAERITech 2003-044, March 2003.

[2] F. J. Sacherer, IEEE Trans. Nucl. Sci. 18, 1105 (1971)

[3] K. R. Crandall et al, "TRACE 3-D Documentation (Third Edition)", LA-UR-97-886, May 1997.

[4] Y. Kondo et al, Proc. 28th Linear Accelerator Meeting in Japan, Tokai, July 2003, TB-2.

[5] T. Ohkawa et al, "Design of the beam transportation line from the LINAC to the $3-\mathrm{GeV}$ RCS for J-PARC" Proc. EPAC2004, Lucerne, July 2004, p.1342. 